Compartmental modeling and identifiability analysis in photophysics: Review Non Peer-reviewed author version

Boens, N. \& AMELOOT, Marcel (2006) Compartmental modeling and identifiability analysis in photophysics: Review. In: INTERNATIONAL JOURNAL OF QUANTUM CHEMISTRY, 106(1). p. 300-315.

DOI: 10.1002/qua.20752

Handle: http://hdl.handle.net/1942/1680 


\section{Compartmental Modeling and Identifiability Analysis in}

\section{Photophysics: A Review}

May 2, 2005

Noël Boens ${ }^{a,{ }^{*}}$ and Marcel Ameloot ${ }^{b}$

${ }^{a}$ Department of Chemistry, Katholieke Universiteit Leuven, Celestijnenlaan 200 F, 3001 Heverlee, Belgium

${ }^{b}$ Biomedisch Onderzoeksinstituut, Limburgs Universitair Centrum, School of Life

Sciences, transnationale Universiteit Limburg, 3590 Diepenbeek, Belgium

*Corresponding author: Noel.Boens@chem.kuleuven.be 


\section{Abstract}

The main objective of this review is to show how the concepts of compartmental modeling can be introduced and applied in photophysics. The term "compartment" in a photophysical context is defined as a subsystem composed of a distinct type of species that acts kinetically in a unique way. Compartments can be divided into ground and excited-state compartments depending upon the state of the composing species. In photophysics, a compartmental system is perturbed by a light pulse (photo-excitation) and its dynamics is followed via fluorescence in the time range from picoseconds to several hundred nanoseconds. In this review, we present the fluorescence $\delta$-response functions for compartmental systems consisting of one excited-state compartment, two reversibly interconnected excited-state compartments, and their corresponding groundstate compartments. In deterministic identifiability one investigates whether the parameters of a specific model can be uniquely defined assuming perfect time-resolved fluorescence data. The identifiability is presented for the model with one excited-state compartment and three models of reversible intermolecular two-state excited-state processes in isotropic environments: (1) model without external quencher, (2) model with added quencher, (3) model with coupled species-dependent rotational diffusion described by Brownian reorientation. The parameters that have to be identified are time-invariant rate constants and parameters related to excitation and emission. It is shown under what conditions the relevant parameters can be identified. For all models, the explicit relationships between the true and alternative model parameters are shown. 


\section{Introduction}

Compartmental modeling is frequently used in biomedicine, pharmacokinetics, analysis of ecosystems, engineering, and chemical reaction kinetics (see, for example, refs 1, 2, 3). Considering this extensive interest, it may seem rather surprising that compartmental modeling of excited-state processes in photophysics has started relatively late. ${ }^{4,5,6,7,8}$ Indeed, as the relaxation of excited-state processes can in many instances be described by a set of coupled first-order differential equations, excited-state systems are formally equivalent with compartmental systems. It will be shown in this review that modeling of excited-state processes in photophysics can conveniently be done within the framework of compartmental analysis.

Let us start by defining what the term "compartment" stands for in a photophysical context. A compartment is a subsystem composed of a distinct type of species that acts kinetically in a unique way. The concentration of the constituting species can change when the compartments exchange material through intramolecular or intermolecular processes. In the perspective of compartmental modeling of excited-state processes, compartments can be divided into ground-state and excited-state compartments depending upon the state of the composing species. The compartmental system then consists of one or more excited-state compartments and their corresponding ground-state compartments. There may be inputs from ground-state compartments into one or more of the excited-state compartments by photo-excitation. There is always output from the excited-state compartments to the ground-state compartments through emission and/or nonradiative deactivation. If the concentrations of the species in the ground state do not significantly change upon photo-excitation, it suffices to consider the excited-state 
compartments only. In that case, the ground-state compartments can be lumped together as the outside environment. Compartments are usually depicted as boxes (rectangles, see Schemes), circles or ovals enclosing the composing species. Single-headed arrows pointing away from a compartment represent outflow from that compartment, whereas single-headed arrows pointing toward a compartment depict inflow into that compartment. In contrast to "classical" compartmental analysis, the terms "size" or "volume" are not relevant for compartments in photophysics.

In kinetics, one is concerned with finding the response of a compartmental system to an external perturbation, given the structure of the system (i.e., the links between the compartments) and the rate constants describing the rates of the intercompartmental transitions. Deriving the kinetic expressions is often referred to as the direct problem of compartmental analysis. In photophysics, the response of the excited-state compartments after photo-excitation (called "the external perturbation" in compartmental jargon) is commonly measured as time-resolved fluorescence. Since fluorescence decay traces can be monitored under a diversity of experimental conditions - the excitation wavelength $\lambda_{i}^{\mathrm{ex}}$, the emission wavelength $\lambda_{j}^{\mathrm{em}}$, the concentration of coreactant $[\mathrm{X}]_{k}$, the concentration of external quencher $[\mathrm{Q}]_{l}$, the temperature, the added buffer, orientation of excitation and emission polarizers, ... can all be varied independently - compartmental systems in photophysics have more experimental axes to study a given problem than in the "classical" areas of application of compartmental modeling. Moreover, a very large number (several thousands) of data points of high quality can be collected from timeresolved fluorescence measurements. This is usually not possible for the "common" 
compartmental systems. Additionally, the prior knowledge available in fluorescence studies might also be different from that in "classical" compartmental systems.

An essential first stage of any identifiability analysis deals with finding solutions for the model parameters when ideal (noise-free) data are available. This identifiability with perfect observations is called deterministic identifiability.

In the deterministic identification (or identifiability) problem, one investigates as to whether or not the parameters of a specific model are uniquely defined under error-free observations, given that the model is completely specified including input into, output from, and exchange between the compartments. ${ }^{1-3}$ The deterministic identification problem reduces to the question of whether a system of nonlinear algebraic equations has a unique solution. There are three possible outcomes to the identifiability analysis.

(1) The parameters of an assumed model can be estimated uniquely and the model is said to be uniquely (globally) identifiable from the idealized experiment.

(2) Any of a finite number of alternative estimates for some or all of the model parameters fits the data and the model is locally identifiable.

(3) An infinite number of model parameter estimates fits the data and the model is unidentifiable from the experiment.

For the linear, time-invariant models with a limited number of excited-state compartments that are generally encountered in photophysics, the parameters that have to be identified are the time-invariant rate constants and parameters related to excitation and emission.

Deterministic identification is thus concerned only with perfect observations and can point the way to improved experimental design. Imperfect data resulting from noisy 
observations sampled over a limited time range affect the accuracy and precision with which model parameters can be estimated. This numerical parameter estimation of the model parameters and the statistical properties of the estimates are the subject of the second stage of any identifiability analysis and is called numerical identifiability. This topic, however, is beyond the scope of this review.

Since the first deterministic identification of an intermolecular two-state excited-state process, identifiability studies of a broad range of compartmental models of excited-state processes have been reported (see ref 9 for literature data). In the current review, we consider the compartmental analysis and the deterministic identifiability of a limited number of photophysical models. We start with the simplest model (with one excitedstate compartment). Next, models for reversible intermolecular two-state excited-state processes in the absence ${ }^{9,10}$ and presence ${ }^{11}$ of quencher as monitored by the total (or "magic angle"-selected) fluorescence are discussed. Finally, the model of a reversible intermolecular two-state excited-state process coupled with species-dependent rotational diffusion described by Brownian reorientation is considered. ${ }^{12,} 13$

The paper is organized as follows. Section 2 starts with some general concepts and definitions of the analysis of compartmental models and their identifiability. Two identification (or identifiability) approaches will be discussed in some detail. In Section 3 , the fluorescence kinetics and identifiability analysis of the system with one excitedstate compartment will be considered to illustrate some aspects of compartmental analysis in photophysics. In Section 4, the fluorescence dynamics and the identifiability analyses of three models of a reversible intermolecular two-state excited-state process without transient effects (i.e., with kinetics governed by time-invariant rate constants) are 
presented. We will discuss what the effect is of the addition of quencher and of analyzing the time-resolved fluorescence anisotropy. When the reversible intermolecular two-state excited-state process is coupled with species-dependent rotational diffusion - described by Brownian reorientation - both spherically and cylindrically symmetric rotors, with no change in the principal axes of rotation in the latter, will be considered. The paper concludes with a discussion of the results of the identifiability studies and their relevance for designing experimental fluorescence decay measurements. Finally, we will refer to some applications of compartmental analysis in photophysics.

For anyone new to the field, this limited review can serve as a low threshold entry point to compartmental modeling in photophysics. To make this introduction to this field as accessible as possible, we have restricted the discussion to simple models. Furthermore, we have avoided unnecessary mathematical derivations that may cloud the essentials. A full mathematical treatment can be found in the literature. ${ }^{9-13}$ Those already familiar with compartmental models and their application (in the areas of biomedicine, pharmacokinetics, ecology, engineering) will, we hope, find that compartmental modeling in photophysics is an exciting rather recent development with many potential applications.

\section{Fluorescence kinetics and identifiability analysis: general concepts}

Given a certain photophysical model, the fluorescence $\delta$-response function $f(t)$ (i.e., after an excitation pulse of infinitely short duration described by the Dirac delta function) is a prerequisite for tackling the important problem of identifiability - that is, whether the unknown model parameters can be recovered from the fluorescence $\delta$-response function $f(t)$. 
If a causal, linear, time-invariant compartmental system consisting of $N$ ground-state compartments is excited with a $\delta$-pulse of low intensity at time zero, so that the groundstate species population is not appreciably depleted, the time course of the excited-state species $\mathbf{x}^{*}(t)$ can be described by the following differential matrix equation:

$$
\frac{\mathrm{d} \mathbf{x}^{*}(t)}{\mathrm{d} t}=\mathbf{A} \mathbf{x}^{*}(t), \quad \mathrm{t} \geq 0
$$

with $\mathbf{x}^{*}$ a $N \times 1$ vector whose elements are the concentrations of the excited-state species:

$$
\mathbf{x}^{*}=\left(\left[1^{*}\right]\left[2^{*}\right] \quad \ldots \quad\left[N^{*}\right]\right)^{\mathrm{T}}
$$

A is a $N \times N$ matrix (called "compartmental matrix" in compartmental parlance and "system matrix" in systems theory) containing the kinetic information ("transfer coefficients") of all processes:

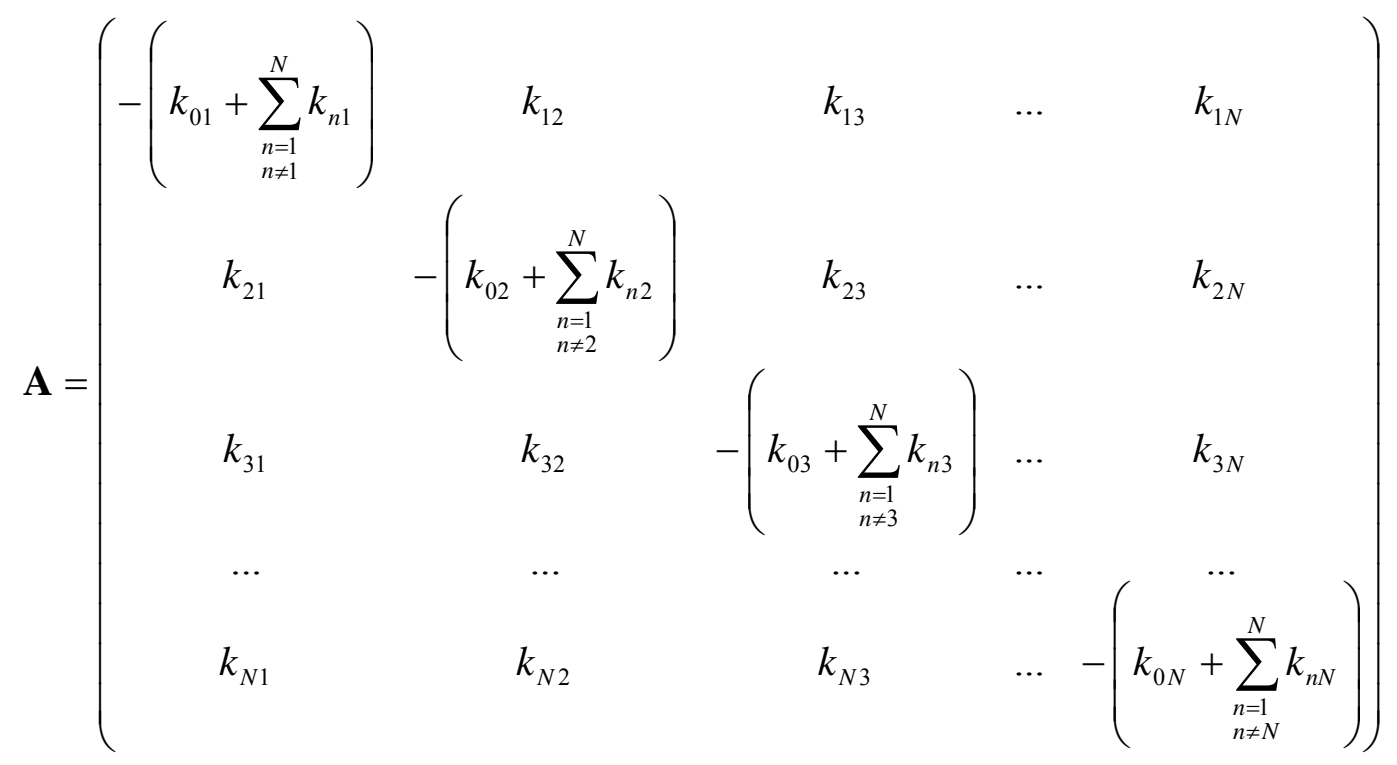

$k_{m n}$ stands for the rate constant of transfer to compartment $m^{*}$ from compartment $n^{*}$; the subscript 0 denotes a ground-state compartment. In photophysics there is always deactivation [through fluorescence $(\mathrm{F})$ emission and/or nonradiative (NR) processes] from the excited-state compartments to the associated ground-state compartments $\left(k_{0 n}>\right.$ 
$0, n=1,2, \ldots, N$ with $\left.k_{0 n}=k_{\mathrm{F} n}+k_{\mathrm{NR} n}\right)$. Therefore, all excited-state compartments are said to be open. Furthermore, all rate constants $k_{m n}(m \neq n)$ and $x_{n}^{*}$ are nonnegative: $k_{m n} \geq 0, m$ $\neq n ; x_{n}^{*} \geq 0, n=1,2, \ldots, N$.

From Eq. (1) we see that the dynamics of compartmental models are simply defined by a set of coupled first-order differential equations.

Photo-excitation with light of wavelength $\lambda_{i}^{\text {ex }}$ thus produces $N$ associated excited-state compartments, for which the fluorescence $\delta$-response function $f(t)$ at emission wavelength $\lambda_{j}^{\mathrm{em}}$ can be expressed in matrix form as ${ }^{10}$

$$
f(t)=\mathbf{c} \mathbf{x}^{*}(t)=\mathbf{c} e^{t \mathrm{~A}} \mathbf{b}=\mathbf{c} \mathbf{U} e^{t \Gamma} \mathbf{U}^{-1} \mathbf{b}
$$

In this equation, we assume that the matrix $\mathbf{A}$ has $N$ linearly independent eigenvectors $\mathbf{U}_{1}$, $\mathbf{U}_{2}, \ldots, \mathbf{U}_{N}$ associated with the eigenvalues $\gamma_{1}, \gamma_{2}, \ldots, \gamma_{N}$, respectively, i.e., $\mathbf{A}=\mathbf{U} \Gamma \mathbf{U}^{-1}$ with $\mathbf{U}=\left[\mathbf{U}_{1}, \mathbf{U}_{2}, \ldots, \mathbf{U}_{N}\right]$ and $\mathbf{U}^{-1}$ the inverse of the matrix of the eigenvectors, $\Gamma$ is the diagonal matrix of $N$ eigenvalues, $\Gamma_{m m}=\gamma_{m}$ and $\Gamma_{m n}=0$ for $m \neq n(m, n=1,2, \ldots, N)$, and $\mathrm{e}^{t \Gamma}=\operatorname{diag}\left[\exp \left(\gamma_{1} t\right), \exp \left(\gamma_{2} t\right), \ldots, \exp \left(\gamma_{N} t\right)\right] . \mathbf{A}, \mathbf{U}$, and $\mathrm{e}^{t \Gamma}$ are functions of the rate constants and for the intermolecular models considered in this paper also of the concentration of coreactant $\mathrm{X}$. The matrix $e^{\mathrm{tA}}$ is called the "transition matrix" in compartmental terminology and is nonnegative for all $t,{ }^{14}$

$$
e^{t \mathbf{A}}=\mathbf{I}+t \mathbf{A}+\frac{(t \mathbf{A})^{2}}{2 !}+\frac{(t \mathbf{A})^{3}}{3 !}+\ldots
$$

with I the $N \times N$ identity matrix. Hence, the fluorescence response of such a compartmental system after perturbation with a light pulse of infinitely short duration consists of a sum of (maximally) $N$ exponentials. 


$$
f(t)=\sum_{n=1}^{N} \alpha_{n} \exp \left(\gamma_{n} t\right)
$$

where $\alpha_{n}$ is the pre-exponential factor associated with the $n$th eigenvalue $\gamma_{n}$ of $\mathbf{A}$. $\mathbf{b}$ is a column vector of dimension $N$ whose (constant nonnegative) elements are the initial (i.e., at time zero) concentrations of each excited-state compartment:

$$
\begin{aligned}
& \mathbf{b}=\left(\begin{array}{llll}
b_{1} & b_{2} & \ldots & b_{N}
\end{array}\right)^{\mathrm{T}} \\
& \mathbf{b}=\mathbf{x}^{*}(0)
\end{aligned}
$$

The elements $b_{n}$ of $\mathbf{b}$ are generally dependent on the excitation wavelength $\lambda_{i}^{\text {ex }}$ and for the intermolecular models considered in this paper also on the concentration of coreactant $X$.

c is a $1 \times N$ vector related to the contribution of each compartment to the emission at $\lambda_{j}^{\mathrm{em}}$ :

$$
\mathbf{c}=\left(\begin{array}{llll}
c_{1 j} & c_{2 j} & \cdots & c_{N j}
\end{array}\right)
$$

The emission weighting factor $c_{m j}$ of species $m^{*}$ at emission wavelength $\lambda_{j}^{\text {em }}$ is given by

$$
C_{m j}=k_{\mathrm{Fm}} \int_{\Delta \lambda_{j}^{\mathrm{em}}} \rho_{m}\left(\lambda_{j}^{\mathrm{em}}\right) \mathrm{d} \lambda_{j}^{\mathrm{em}}
$$

In Eq. (9), $k_{\mathrm{Fm}}$ stands for the fluorescence rate constant of $m^{*} ; \Delta \lambda_{j}^{\mathrm{em}}$ is the emission wavelength interval around $\lambda_{j}^{\mathrm{em}}$ where the fluorescence signal is monitored; $\rho_{m}\left(\lambda_{j}^{\mathrm{em}}\right)$ is the emission density of $m^{*}$ at $\lambda_{j}^{\text {em }}$ defined by

$$
\rho_{m}\left(\lambda_{j}^{\mathrm{em}}\right)=F_{m}\left(\lambda_{j}^{\mathrm{em}}\right) / \int_{\text {full emission band }} F_{m}\left(\lambda_{j}^{\mathrm{em}}\right) \mathrm{d} \lambda_{j}^{\mathrm{em}}
$$


where the integration extends over the whole steady-state fluorescence spectrum $F_{m}\left(\lambda_{j}^{\mathrm{em}}\right)$ of species $m^{*}$. The system $(\mathbf{A}, \mathbf{b}, \mathbf{c})$ that has $f(t)$ as its impulse response function is called a realization of $f(t)$.

Given the realization (A, b, c), the fluorescence $\delta$-response function $f(t)$ is uniquely determined. However, given $f(t)$, it is usually possible to find several realizations $(\mathbf{A}, \mathbf{b}, \mathbf{c})$ that generate the function $f(t)$. A general way of formulating the identification problem is as follows: is it possible to find different realizations of $f(t)$, say $(\mathbf{A}, \mathbf{b}, \mathbf{c})$ and $\left(\mathbf{A}^{+}, \mathbf{b}^{+}, \mathbf{c}^{+}\right)$, so that Eq. (11) holds

$$
f(t, \mathbf{A}, \mathbf{b}, \mathbf{c})=f\left(t, \mathbf{A}^{+}, \mathbf{b}^{+}, \mathbf{c}^{+}\right)
$$

In other words, the fluorescence $\delta$-response function $f(t)$ should be the same for the true $(\mathbf{A}, \mathbf{b}, \mathbf{c})$ and the alternative $\left(\mathbf{A}^{+}, \mathbf{b}^{+}, \mathbf{c}^{+}\right)$model parameter set. ${ }^{2,3}$ Global (unique) identifiability is attained when $\mathbf{A}^{+}=\mathbf{A}, \mathbf{b}^{+}=\mathbf{b}$, and $\mathbf{c}^{+}=\mathbf{c}$ (i.e., a unique set of model parameters is obtained). The model is locally identifiable when there is a limited set of alternative $\mathbf{A}^{+}, \mathbf{b}^{+}$, and $\mathbf{c}^{+}$. An unidentifiable model is found when there are an infinite number of alternative $\mathbf{A}^{+}, \mathbf{b}^{+}$, and $\mathbf{c}^{+}$. The formulation as given by Eq. (11) is suitable for most systems found in biomedicine, pharmacokinetics, ecosystem modeling and engineering, but is inappropriate for photophysical systems where absolute values for $\mathbf{b}$ and c cannot be obtained. Therefore, in a photophysical context, it is more appropriate to use normalized vectors $\widetilde{\mathbf{b}}$ and $\widetilde{\mathbf{c}}^{9,10}$ (see Section 4).

There are several methods available for the analysis of the deterministic identifiability (i.e., identification with perfect, errorless data) of linear, time-invariant models.

In some cases, Eq. (11) can be used directly to determine the relationship between the true and alternative model parameters [see Eq. (20) in Section 3]. 
The approach which, in our investigations of the deterministic identifiability of timeinvariant models for time-resolved fluorescence, has predominantly been used previously ${ }^{10-12}$ involves Markov parameters $m_{m}$ of the realization $(\mathbf{A}, \mathbf{b}, \mathbf{c})$ and elementary symmetric functions $\sigma_{n}$ in $\gamma_{1}, \gamma_{2}, \ldots, \gamma_{N}$. The set of eigenvalues $\gamma_{n}$ of $\mathbf{A}$ allows the construction of functions $\sigma_{n}(n=1,2, \ldots, N)$

$$
\sigma_{1} \equiv \sum_{n} \gamma_{n}, \sigma_{2} \equiv \sum_{m<n} \gamma_{m} \gamma_{n}, \ldots, \sigma_{N} \equiv \gamma_{1} \gamma_{2} \ldots \gamma_{N}
$$

This set of nonlinear equations $\sigma_{n}$ relates the eigenvalues $\gamma_{n}$ or, equivalently, the relaxation times $\tau_{n}=-\gamma_{n}^{-1}$ to the elements of matrix $\mathbf{A}$.

The vectors $\mathbf{b}$ and $\mathbf{c}$ appear only in the Markov parameters $m_{m}(m=0,1, \ldots, N-1)$. The Markov parameters $m_{m}$ are defined as the $m$ th time derivatives of $f(t)$ at time zero:

$$
\begin{aligned}
& m_{m} \equiv \frac{\partial^{m} f(0)}{\partial t^{m}}, \quad m=0,1, \ldots, N-1 \\
& m_{m}=\sum_{n=1}^{N} \alpha_{n} \gamma_{n}^{m}, \quad m=0,1, \ldots, N-1
\end{aligned}
$$

where $\gamma_{n}^{m}$ denotes the $m$ th power of the eigenvalue $\gamma_{n}$ and $\alpha_{n}$ is the associated preexponential factor (see Section 4). In terms of the realization (A, b, c) of $f(t)$, the Markov parameters are expressed by:

$$
m_{m}=\mathbf{c} \mathbf{A}^{m} \mathbf{b}, \quad m=0,1, \ldots, N-1
$$

where $\mathbf{A}^{m}$ represents the $m$ th power of matrix $\mathbf{A}$. For an $N$-compartmental system, only $N$ independent Markov parameters can be constructed for each set of experimental conditions. The identifiability analysis deals with the determination of the unknown elements of matrix $\mathbf{A}$ and vectors $\mathbf{b}$ and $\mathbf{c}$ from $\sigma_{n}$ and $m_{m}$. 
An excellent method of finding a different (alternative) realization $\left(\mathbf{A}^{+}, \mathbf{b}^{+}, \mathbf{c}^{+}\right)$of $f(t)$ is via similarity transformation, ${ }^{2,3,15,16}$ yielding

$$
\mathbf{T} \mathbf{A}^{+}=\mathbf{A} \mathbf{T}
$$

where $\mathbf{T}$ is a constant invertible (or nonsingular) matrix (i.e., det $\mathbf{T} \neq 0$ ) having the same dimension as $\mathbf{A}$.

The alternative $\mathbf{b}^{+}$and $\mathbf{c}^{+}$are given by

$$
\begin{aligned}
\mathbf{b}^{+} & =\mathbf{T}^{-1} \mathbf{b} \\
\mathbf{c}^{+} & =\mathbf{c} \mathbf{T}
\end{aligned}
$$

The major advantage of the similarity transformation approach is that it not only offers a direct way of determining if a model is uniquely or locally identifiable or unidentifiable, but it also gives the explicit relationships between the true and alternative model parameters.

Equations (15-17) should be satisfied for each experimental condition. For the models considered, the possible experimental variables are excitation wavelength $\lambda_{i}^{\text {ex }}$, emission wavelength $\lambda_{j}^{\mathrm{em}}$, coreactant concentration $[\mathrm{X}]_{k}$, and quencher concentration $[\mathrm{Q}]_{l}$. This implies that matrix $\mathbf{T}$ should be independent of $\lambda_{i}^{\mathrm{ex}}, \lambda_{j}^{\mathrm{em}},[\mathrm{X}]_{k}$, and $[\mathrm{Q}]_{l}$.

\section{Fluorescence kinetics and identifiability analysis of the compartmental system with one excited-state compartment}

The two objectives of this section are (1) to introduce as smoothly as possible the general concepts of compartmental analysis into the field of photophysics and (2) to demonstrate that even for the simplest model there are restrictions on the amount of information that can be recovered from perfect time-resolved fluorescence data. 
In this section, a linear, time-invariant compartmental system consisting of one groundstate species (symbolized by 1) as shown in Scheme 1 is considered. Photo-excitation produces the excited-state species $1^{*}$ which can return to its ground state 1 with rate constant $k_{01}=k_{\mathrm{F} 1}+k_{\mathrm{NR} 1}$. This rate constant is required to be positive $\left(k_{01}>0\right)$. Scheme 1 is a graphical illustration of a photophysical system comprising one ground-state and one excited-state compartment. The system with one excited-state compartment is the scalar case of the compartmental model.

\section{Insert Scheme $1+$ caption}

The differential equation describing the time-course of the quantity $x_{1}^{*}=\left[1^{*}\right]$ in the excited-state compartment after $\delta$-pulse photo-excitation at $\lambda_{i}^{\text {ex }}$ is

$$
\frac{\mathrm{d} x_{1}^{*}(t)}{\mathrm{d} t}=-k_{01} x_{1}^{*}(t)
$$

The fluorescence $\delta$-response function $f_{i j}(t)$ is

$$
f_{i j}(t)=b_{1} c_{1} \exp \left(-k_{01} t\right)
$$

The subscripts $i$ and $j$ on $f_{i j}(t)$ indicate its dependence on $\lambda_{i}^{\mathrm{ex}}$ and $\lambda_{j}^{\mathrm{em}}$, respectively. In photophysics, one observes the change of concentration of excited species $1^{*}$ via the measurement of the time-resolved fluorescence signal. This signal is dependent on the experimental observables $\left\{\lambda_{i}^{\mathrm{ex}}, \lambda_{j}^{\mathrm{em}}, t\right\}$ and the model parameters $\left\{k_{01}, b_{1}, \mathrm{c}_{1}\right\}$.

The question one would like to answer first in identifiability analysis, is whether it is possible to obtain an alternative realization $\left\{k_{01}^{+}, b_{1}^{+}, c_{1}^{+}\right\}$of $f_{i j}(t)$ different from the true model parameter set $\left\{k_{01}, b_{1}, c_{1}\right\}$, so that $f_{i j}(t)$ is the same for the true and alternative realization: 


$$
f_{i j}\left(t, k_{01}, b_{1}, c_{1}\right)=f_{i j}\left(t, k_{01}^{+}, b_{1}^{+}, c_{1}^{+}\right)
$$

Equation (20) is the specific form of Eq. (11) for a system with one excited-state compartment. Rewriting Eq. (20) specifically for the considered model, one has

$$
b_{1} c_{1} \exp \left(-k_{01} t\right)=b_{1}^{+} c_{1}^{+} \exp \left(-k_{01}^{+} t\right)
$$

Evaluating Eq. (21) at time zero gives

$$
b_{1}^{+} c_{1}^{+}=b_{1} c_{1}
$$

and hence

$$
k_{01}^{+}=k_{01}
$$

This demonstrates that the rate constant $k_{01}$ is uniquely identified, as is the product $b_{1} c_{1}$. However, the individual $b_{1}$ and $c_{1}$ cannot be identified. Indeed, from Eq. (16) with $\mathbf{T}=$ $\left(t_{1}\right)$ we have $b_{1}^{+}=b_{1} / t_{1}$ and from Eq. (17) we obtain $c_{1}^{+}=c_{1} t_{1}$, implying that there are an infinite number of alternative $b_{1}^{+}$and $c_{1}^{+}$. Although the composite rate constant $k_{01}$ is uniquely identified, the composing rate constants $k_{\mathrm{F} 1}$ and $k_{\mathrm{NR} 1}$ are not identifiable without extra (prior) information. This additional knowledge is available if the fluorescence quantum yield $\phi_{\mathrm{f}}$ is known:

$$
\phi_{\mathrm{f}}=k_{\mathrm{F} 1} / k_{01}
$$

The unique values of $k_{\mathrm{F} 1}$ and $k_{\mathrm{NR} 1}$ are then calculated according to

$$
\begin{aligned}
& k_{\mathrm{F} 1}=\phi_{\mathrm{f}} k_{01} \\
& k_{\mathrm{NR} 1}=k_{01}\left(1-\phi_{\mathrm{f}}\right)
\end{aligned}
$$

This simple model shows that only the rate constant $k_{01}$ is uniquely identified. If one wants to know the contributions to $k_{01}$ of fluorescence $\left(k_{\mathrm{F} 1}\right)$ and nonradiative decay $\left(k_{\mathrm{NR} 1}\right)$, prior knowledge is needed. 


\section{Fluorescence kinetics and identifiability analysis of models of reversible intermolecular two-state excited-state process}

\section{A. Model without external quencher}

\section{Insert Scheme 2 + caption}

In this section, we will start with the fluorescence kinetics of an intermolecular system consisting of two distinct interchanging ground-state species (1 and 2) and two corresponding excited-state species $\left(1^{*}\right.$ and $\left.2^{*}\right)$ as depicted in Scheme 2 (i.e., two groundstate compartments and two associated excited-state compartments). Ground-state species 1 can reversibly react with coreactant $\mathrm{X}$ to form ground-sate species 2. Photo-excitation creates the excited-state species $1^{*}$ and $2^{*}$, which decay by rate constants $k_{01}$ and $k_{02}$ to their respective ground states. The rate constant describing the intermolecular transformation of $1^{*}$ into $2^{*}$ is represented by $k_{21}$, while the reverse process is described by $k_{12}$. These rate constants are required to be positive as we deal with a reversible excited-state process.

The fluorescence impulse response function $f_{i j k}(t)$ [Eq. (4)] for the photophysical system shown in Scheme 2 is explicitly given by

$$
f_{i j k}(t)=\mathbf{c}_{j} \exp \left(t \mathbf{A}_{k}\right) \mathbf{b}_{i k}
$$

The subscripts $i, j$, and $k$ in Eq. (25) refer to the excitation wavelength $\lambda_{i}^{\text {ex }}$, the emission wavelength $\lambda_{j}^{\mathrm{em}}$, and the coreactant concentration $[\mathrm{X}]_{k}$, respectively

Matrix $\mathbf{A}_{k}$ is given by

$$
\mathbf{A}_{k}=\left(\begin{array}{cc}
-\left(k_{01}+k_{21}[\mathrm{X}]_{k}\right) & k_{12} \\
k_{21}[\mathrm{X}]_{k} & -\left(k_{02}+k_{12}\right)
\end{array}\right)
$$

The $2 \times 1$ vector $\mathbf{b}_{i k}$ contains the initial concentrations of excited species $1^{*}$ and $2^{*}$ : 


$$
\mathbf{b}_{i k}=\left(\begin{array}{ll}
b_{1 i k} & b_{2 i k}
\end{array}\right)^{\mathrm{T}}
$$

with $b_{n i k}=\left[n^{*}\right]_{0}$ at $\lambda_{i}^{\mathrm{ex}}$ and $[\mathrm{X}]_{k}$.

$\mathbf{c}_{j}$ is the $1 \times 2$ vector of the emission weighting factors $c_{m j}(m=1,2)$ at emission wavelength $\lambda_{j}^{\text {em }}$ defined by Eq. (9).

$$
\mathbf{c}_{j}=\left(\begin{array}{ll}
c_{1 j} & c_{2 j}
\end{array}\right)
$$

Equation (25) can be written in the common bi-exponential format:

$$
f_{i j k}(t)=\alpha_{1} \exp \left(\gamma_{1} t\right)+\alpha_{2} \exp \left(\gamma_{2} t\right) \quad t \geq 0
$$

The eigenvalues $\gamma_{1,2}$ of the $2 \times 2$ matrix $\mathbf{A} \equiv\left(a_{m n}\right)$ are

$$
\gamma_{1,2}=\frac{1}{2}\left[a_{11}+a_{22} \pm \sqrt{\left(a_{11}-a_{22}\right)^{2}+4 a_{12} a_{21}}\right]
$$

For the considered model, the elements $a_{m n}$ of $\mathbf{A}$ are shown in Eq. (26). Since the part under the square root in Eq. (30) cannot be negative, the eigenvalues $\gamma_{1,2}$ must be real. For a reversible two-state excited-state process, the eigenvalues $\gamma_{1,2}$ are distinct. Repeated eigenvalues are obtained if the part under the square root in Eq. (30) is zero. For this, $a_{11}$ and $a_{22}$ must be equal and $a_{12} a_{21}$ must be zero; the latter implies that either $a_{12}$ or $a_{21}$ must be zero. In that case the excited-state process would not be reversible anymore. If $a_{12}$ and $a_{21}$ were both zero, the compartments would not be connected.

The pre-exponentials $\alpha_{1,2}$ corresponding to $\gamma_{1,2}$ are

$$
\begin{aligned}
& \alpha_{1}=c_{1} \beta_{11}+c_{2} \beta_{21} \\
& \alpha_{2}=c_{1} \beta_{12}+c_{2} \beta_{22} \\
& \beta_{11}=\frac{b_{1}\left(\gamma_{2}-a_{11}\right)-b_{2} a_{12}}{\gamma_{2}-\gamma_{1}}
\end{aligned}
$$




$$
\begin{aligned}
& \beta_{12}=-\frac{b_{1}\left(\gamma_{1}-a_{11}\right)-b_{2} a_{12}}{\gamma_{2}-\gamma_{1}} \\
& \beta_{21}=\frac{b_{2}\left(\gamma_{2}-a_{22}\right)-b_{1} a_{21}}{\gamma_{2}-\gamma_{1}} \\
& \beta_{22}=-\frac{b_{2}\left(\gamma_{1}-a_{22}\right)-b_{1} a_{21}}{\gamma_{2}-\gamma_{1}}
\end{aligned}
$$

To keep Eqs. (30-32) transparent, we did not use the subscripts $i, j$, and $k$ on $b, c, \alpha, \beta$, and $\gamma$ to indicate their dependence on $\lambda_{i}^{\mathrm{ex}}, \lambda_{j}^{\mathrm{em}}$, and $[\mathrm{X}]_{k}$. For the considered model the eigenvalues $\gamma_{1,2}$ [Eq. (30)] depend on the rate constants and $[\mathrm{X}]_{k}$, while the preexponentials $\alpha_{1,2}$ [Eq. (31)] depend additionally on $\lambda_{i}^{\mathrm{ex}}$ (through $\mathbf{b}_{i k}$ ) and $\lambda_{j}^{\mathrm{em}}$ (through $\left.\mathbf{C}_{j}\right)$.

Now let us investigate the identifiability via similarity transformation. For the considered system with two excited-state compartments, the $2 \times 2$ matrix $\mathbf{T}$ is

$$
\mathbf{T}=\left(\begin{array}{ll}
t_{1} & t_{2} \\
t_{3} & t_{4}
\end{array}\right)
$$

Four rate constants $\left(k_{01}, k_{21}, k_{02}, k_{12}\right)$ have to be identified. The matrix multiplication in Eq. (15) with A given by Eq. (26) leads to two sets of rate constant values.

Set I is the original set: $k_{01}^{+}=k_{01}, k_{12}^{+}=k_{12}, k_{21}^{+}=k_{21}, k_{02}^{+}=k_{02}$ with $\mathbf{T}=t_{1} \mathbf{I}$, with $\mathbf{I}$ the $2 \times 2$ identity matrix and where $k_{m n}^{+}$represents the alternative value of $k_{m n}$.

Set II is the alternative set: $k_{01}^{+}=k_{02}+k_{12}, k_{12}^{+}=k_{01}-k_{02}, k_{21}^{+}=k_{21}, k_{02}^{+}=k_{02}$ with $\mathbf{T}$ given by Eq. (34) with $t_{3} / t_{1}=\left(k_{01}-k_{02}-k_{12}\right) / k_{12}$ :

$$
\mathbf{T}=\left[\begin{array}{cc}
t_{1} & 0 \\
t_{3} & t_{1}+t_{3}
\end{array}\right]
$$


Since $k_{12}^{+}$must be positive, because a reversible excited-state process is considered, set II is possible only if $k_{01}>k_{02}$. If $k_{01}<k_{02}$, the true set I of rate constants is recovered.

The above results are in perfect agreement with the results obtained via the deterministic identification analysis based on elementary functions $\sigma_{n}[n=1,2$, Eqs. $(35,36)]$ :

$$
\begin{aligned}
& \sigma_{1 k} \equiv \gamma_{1 k}+\gamma_{2 k}=a_{11, k}+a_{22} \\
& \sigma_{1 k}=-\left(k_{01}+k_{21}[\mathrm{X}]_{k}+k_{02}+k_{12}\right) \\
& \sigma_{2 k} \equiv \gamma_{1 k} \gamma_{2 k}=a_{11, k} a_{22}-a_{12} a_{21, k} \\
& \sigma_{2 k}=k_{01}\left(k_{02}+k_{12}\right)+k_{02} k_{21}[\mathrm{X}]_{k}
\end{aligned}
$$

The matrix multiplication in Eqs. (16) and (17) for $\mathbf{T}=t_{1} \mathbf{I}$ (set I) indicates that the alternative $\mathbf{b}_{i k}^{+}$and $\mathbf{c}_{j}^{+}$are only known up to the scaling factor $t_{1}$. This means that the photophysical system expressed in terms of $\mathbf{A}, \mathbf{b}_{i k}$, and $\mathbf{c}_{j}$ is unidentifiable. Since the vectors $\mathbf{b}_{i k}$ and $\mathbf{c}_{j}$ are experimentally not accessible from time-resolved fluorescence experiments, it is more appropriate to use normalized vectors $\widetilde{\mathbf{b}}_{i k}$ and $\widetilde{\mathbf{c}}_{j}$. The elements $\widetilde{b}_{n i k}$ of $\widetilde{\mathbf{b}}_{i k}$ and $\widetilde{c}_{j}$ of $\widetilde{\mathbf{c}}_{j}$ are defined as

$$
\begin{array}{ll}
\widetilde{b}_{n i k} \equiv b_{n i k} /\left(b_{1 i k}+b_{2 i k}\right) & n=1,2 \\
\widetilde{c}_{m j} \equiv c_{m j} /\left(c_{1 j}+c_{2 j}\right) & m=1,2
\end{array}
$$

Use of normalized $\widetilde{b}_{1 i k}^{+}$and $\widetilde{b}_{1 i k}$ in Eq. (16), and $\widetilde{c}_{1 j}^{+}$and $\widetilde{c}_{1 j}$ in Eq. (17) leads to $\widetilde{b}_{1 i k}^{+}=\widetilde{b}_{1 i k}$ and $\widetilde{c}_{1 j}^{+}=\widetilde{c}_{1 j}$. 
Using normalized spectral parameters $\widetilde{b}_{1 i k}\left(0 \leq \widetilde{b}_{1 i k} \leq 1\right)$ and $\widetilde{c}_{1 j}\left(0 \leq \widetilde{c}_{1 j} \leq 1\right)$ in global compartmental analysis of related fluorescence decay traces allows one to link $\widetilde{b}_{1 i k}$ at the same $\lambda_{i}^{\mathrm{ex}}$ and $[\mathrm{X}]_{k}$, whereas $\widetilde{c}_{1 j}$ can be linked at the same $\lambda_{j}^{\mathrm{em}}$.

Now we consider the identifiability of $\widetilde{b}_{1 i k}$ and $\widetilde{c}_{1 j}$ associated with set II of the rate constants, where matrix $\mathbf{T}$ is given by Eq. (34). As was the case for set $\mathrm{I}, t_{1}$ cannot be determined for set II and consequently the model expressed in terms of $\mathbf{A}, \mathbf{b}_{i k}$, and $\mathbf{c}_{j}$ is unidentifiable. Use of normalized $\widetilde{b}_{n i k}^{+}$and $\widetilde{c}_{n}^{+}$and the fact that they should be restricted to the range $[0,1]$ allows one to define ranges of physically acceptable $\widetilde{b}_{n i k}^{+}$and $\widetilde{c}_{m j}^{+}$ values.

Although the similarity transformation approach expresses the explicit relationships between the true and alternative model parameters, it does not allow one to determine how many concentrations $[\mathrm{X}]$ and emission wavelenghts $\lambda^{\mathrm{em}}$ are necessary for identifiability in terms of the rate constants and the normalized $\widetilde{\mathbf{b}}_{i k}$ and $\widetilde{\mathbf{c}}_{j}$. In the following we will use Markov parameters $m_{m}(m=0,1)$ to answer this question. $m_{0, j k}$ is given by Eq. (39), while $m_{1, i j k}$ is expressed as a function of the matrix elements of $\mathbf{A}_{k}$ in Eq. (40) and as a function of the rate constants and $[\mathrm{X}]_{k}$ in Eq. (41):

$$
\begin{aligned}
& m_{0, j i j k}=\mathbf{c}_{j} \mathbf{b}_{i k}=c_{1 j} b_{1 i k}+c_{2 j} b_{2 i k} \\
& m_{1, j i k}=\mathbf{c}_{j} \mathbf{A}_{k} \mathbf{b}_{i k}=b_{1 i k}\left(c_{1 j} a_{11, k}+c_{2 j} a_{21, k}\right)+b_{2 i k}\left(c_{1 j} a_{12}+c_{2 j} a_{22}\right) \\
& m_{1, j i k}=-b_{1 i k} c_{1 j}\left(k_{01}+k_{21}[\mathrm{X}]_{k}\right)+b_{1 i k} c_{2 j} k_{21}[\mathrm{X}]_{k}+b_{2 i k} c_{1 j} k_{12}-b_{2 i k} c_{2 j}\left(k_{02}+k_{12}\right)
\end{aligned}
$$

Use of normalized $\widetilde{\mathbf{b}}_{i k}$ and $\widetilde{\mathbf{c}}_{j}$ allows one to rewrite the above equations with $\kappa_{i j k}$ as a scaling constant. 


$$
\begin{aligned}
& m_{0, i j k}=\kappa_{i j k} \widetilde{\mathbf{c}}_{j} \widetilde{\mathbf{b}}_{i k}=\kappa_{i j k}\left(\widetilde{c}_{1 j} \widetilde{b}_{1 i k}+\widetilde{c}_{2 j} \widetilde{b}_{2 i k}\right) \\
& m_{1, i j k}=\kappa_{i j k} \widetilde{\mathbf{c}}_{j} \mathbf{A}_{k} \widetilde{\mathbf{b}}_{i k}=\kappa_{i j k}\left[\widetilde{b}_{1 i k}\left(\widetilde{c}_{1 j} a_{11, k}+\widetilde{c}_{2 j} a_{21, k}\right)+\widetilde{b}_{2 i k}\left(\widetilde{c}_{1 j} a_{12}+\widetilde{c}_{2 j} a_{22}\right)\right] \\
& m_{1, i j k}=\kappa_{i j k}\left[-\widetilde{b}_{1 i k} \widetilde{c}_{1 j}\left(k_{01}+k_{21}[\mathrm{X}]_{k}\right)+\widetilde{b}_{1 i k} \widetilde{c}_{2 j} k_{21}[\mathrm{X}]_{k}+\widetilde{b}_{2 i k} \widetilde{c}_{1 j} k_{12}-\widetilde{b}_{2 i k} \widetilde{c}_{2 j}\left(k_{02}+k_{12}\right)\right]
\end{aligned}
$$

It has been shown via the symbolic mathematics program MAPLE V (Waterloo Maple Inc.) that six decay traces - measured at two coreactant concentrations $[\mathrm{X}]$ and three emission wavelengths $\lambda^{\mathrm{em}}$ (corresponding to different $\widetilde{c}_{1}$ ) - are required for the model to be locally identifiable in terms of the rate constants, $\widetilde{b}_{1}$ and $\widetilde{c}_{1}$. Two sets of rate constants and concomitant spectral parameters $\widetilde{b}_{1 i k}$ and $\widetilde{c}_{1 j}$ may be found. For set I, we have $\widetilde{b}_{1 i k}^{+}=\widetilde{b}_{1 i k}$ and $\widetilde{c}_{1 j}^{+}=\widetilde{c}_{1 j}$. For set II, the admissible $\widetilde{b}_{1 i k}^{+}$and $\widetilde{c}_{1 j}^{+}$can be expressed as a function of $\widetilde{b}_{1 i k}, \widetilde{c}_{1 j}$, and the rate constants.

\section{B. Model with added quencher}

\section{Insert Scheme $3+$ caption}

Consider the molecular system (Scheme 3) with an equilibrium between two different species 1 and 2 in the ground state which form upon photo-excitation the excited-state species $1^{*}$ and $2^{*}$, respectively. The rate constants $k_{01}, k_{02}, k_{21}$, and $k_{12}$ have the same meaning as in Scheme 2. By addition of an external quencher, Q, with concentration $[\mathrm{Q}]_{l}$, the depletion of the excited states is enhanced by $k_{\mathrm{q} 1}[\mathrm{Q}]_{l}$ for $1^{*}$ and $k_{\mathrm{q} 2}[\mathrm{Q}]_{l}$ for $2^{*}$. It is assumed that the quencher Q has only an effect on the excited species and does not affect the ground-state equilibrium. The fluorescence $\delta$-response function $f_{i j k l}(t)$ can be expressed in matrix notation:

$$
f_{i j k l}(t)=\mathbf{c}_{j} \exp \left(t \mathbf{A}_{k l}\right) \mathbf{b}_{i k}
$$


The subscripts $i, j, k$ and $l$ in Eq. (45) refer to $\lambda_{i}^{\mathrm{ex}}, \lambda_{j}^{\mathrm{em}},[\mathrm{X}]_{k}$, and [Q $]_{l}$, respectively.

Matrix $\mathbf{A}_{k l}$ is given by

$$
\mathbf{A}_{k l}=\left(\begin{array}{cc}
-\left(k_{01}+k_{21}[\mathrm{X}]_{k}+k_{\mathrm{q} 1}[\mathrm{Q}]_{l}\right) & k_{12} \\
k_{21}[\mathrm{X}]_{k} & -\left(k_{02}+k_{12}+k_{\mathrm{q} 2}[\mathrm{Q}]_{l}\right)
\end{array}\right)
$$

$\mathbf{b}_{i k}$ and $\mathbf{c}_{j}$ are given by Eqs. (27) and (28), respectively. As it is assumed that Q does not affect the ground-state equilibrium, $\mathbf{b}_{i k}$ is independent of $[\mathrm{Q}]_{l}$.

$f_{i j k l}(t)$ can be expressed in the common bi-exponential format [Eq. (29)], with the eigenvalues $\gamma_{1,2}$ [Eq. (30)] dependent on the rate constants, $[\mathrm{X}]_{k}$, and $[\mathrm{Q}]_{l}$. The preexponentials $\alpha_{1,2}$ [Eq. (31)] are dependent additionally on $\lambda_{i}^{\mathrm{ex}}$ and $\lambda_{j}^{\mathrm{em}}$.

First, we investigate the identifiability via similarity transformation. Six rate constants $\left(k_{01}, k_{21}, k_{02}, k_{12}, k_{\mathrm{q} 1}, k_{\mathrm{q} 2}\right)$ have to be determined. Matrix $\mathbf{T}$ has the same dimension as $\mathbf{A}$ and is given by Eq. (33). If the rate constants of quenching are different $\left(k_{\mathrm{q} 1} \neq k_{\mathrm{q} 2}\right)$, the matrix multiplication in Eq. (15) leads to the unique set of original rate constants (i.e., set I of Section 4A): $k_{01}^{+}=k_{01}, k_{12}^{+}=k_{12}, k_{21}^{+}=k_{21}, k_{02}^{+}=k_{02}$, and $k_{\mathrm{q} 1}^{+}=k_{\mathrm{q} 1}, k_{\mathrm{q} 2}^{+}=k_{\mathrm{q} 2}$ with $\mathbf{T}$ $=t_{1}$ I. If the quenching rate constants are identical $\left(k_{\mathrm{q} 1}=k_{\mathrm{q} 2}\right)$ and additionally if $k_{01}>k_{02}$, the alternative set of rate constants is given by set II of Section $4 \mathrm{~A}: k_{01}^{+}=k_{02}+k_{12}$, $\left.k_{12}^{+}=k_{01}-k_{02}, k_{21}^{+}=k_{21}, k_{02}^{+}=k_{02}\right)$ and $k_{\mathrm{q} 1}^{+}=k_{\mathrm{q} 2}^{+}=k_{\mathrm{q} 1}=k_{\mathrm{q} 2}$.

Use of normalized $\widetilde{b}_{1 i k}$ and $\widetilde{c}_{1 j}$ for $\mathbf{T}=t_{1} \mathbf{I}$ leads to the unique spectral parameters: $\widetilde{b}_{1 i k}^{+}=\widetilde{b}_{1 i k}$ and $\widetilde{c}_{1 j}^{+}=\widetilde{c}_{1 j}$.

To conclude, if the quenching rate constants of a quencher added to a reversible intermolecular two-state excited-state process are different $\left(k_{\mathrm{q} 1} \neq k_{\mathrm{q} 2}\right)$, this model is uniquely identifiable in terms of rate constants and normalized $\widetilde{b}_{1 i k}$ and $\widetilde{c}_{1 j}$ 
Second, the identifiability of the six rate constants can be done using $\sigma_{1 k l}$ and $\sigma_{2 k l}$ [Eq. (47)]:

$$
\begin{aligned}
& \sigma_{1 k l}=-\left(k_{01}+k_{02}+k_{12}\right)-k_{21}[\mathrm{X}]_{k}-\left(k_{\mathrm{q} 1}+k_{\mathrm{q} 2}\right)[\mathrm{Q}]_{l} \\
& \sigma_{2 k l}=k_{01}\left(k_{02}+k_{12}\right)+k_{02} k_{21}[\mathrm{X}]_{k}+\left\{\left(k_{01}+k_{21}[\mathrm{X}]_{k}\right) k_{\mathrm{q} 2}+\left(k_{02}+k_{12}\right) k_{\mathrm{q} 1}\left\{[\mathrm{Q}]_{l}+\right.\right. \\
& k_{\mathrm{q} 1} k_{\mathrm{q} 2}[\mathrm{Q}]^{2}
\end{aligned}
$$

For each combination $\left([\mathrm{X}]_{k},[\mathrm{Q}]_{l}\right)$, one has one set of the above equations. It has been shown that four decay traces - measured at two coreactant concentrations $[\mathrm{X}]$ and two quencher concentrations $[\mathrm{Q}]$, namely $\left\{\left([\mathrm{X}]_{1},[\mathrm{Q}]_{1}\right),\left([\mathrm{X}]_{1},[\mathrm{Q}]_{2}\right),\left([\mathrm{X}]_{2},[\mathrm{Q}]_{1}\right),\left([\mathrm{X}]_{2},[\mathrm{Q}]_{2}\right)\right\}$

- lead to the identification of all six rate constants, provided that $k_{\mathrm{q} 1} \neq k_{\mathrm{q} 2}: k_{01}^{+}=k_{01}$, $k_{12}^{+}=k_{12}, k_{21}^{+}=k_{21}, k_{02}^{+}=k_{02}, k_{\mathrm{q} 1}^{+}=k_{\mathrm{q} 1}, k_{\mathrm{q} 2}^{+}=k_{\mathrm{q} 2}$. For more details, we refer to the original paper.

\section{C. Model with species-dependent rotational diffusion}

\section{Insert Figure $1+$ caption}

The photophysical system consisting of two different interchanging species 1 and 2, each with distinct rotational characteristics - as depicted in Figure 1 - is considered. All the rate constants are assumed independent of the instantaneous orientation of the species. The rotational relaxation of each excited-state species is governed by its principal rotational diffusion constants, here $D_{\perp}$ and $D_{\|}$for rotation, respectively, of and about the symmetry axis of each of the cylindrically symmetric rotors depicted in Figure 1 . The fluorescence impulse response function $I_{\| i j k}(t)$ for the plane-polarized component of emission of the two excited states $1^{*}$ and $2^{*}$, having its electric vector polarized parallel to the electric vector of the plane-polarized excitation light, and the fluorescence $\delta$-response 
function $I_{\perp i j k}(t)$ for the perpendicularly polarized component can be expressed, in the case of pure transitions and isotropic solutions, as: ${ }^{17}$

$$
\begin{aligned}
& I_{\|_{i k j}}(t)=\frac{1}{3} S_{i k j}(t)\left[1+2 r_{i k j}(t)\right]=\frac{1}{3} S_{i k j}(t)+\frac{2}{3} D_{i k j}(t) \\
& I_{\perp i k j}(t)=\frac{1}{3} S_{i k j}(t)\left[1-r_{i k j}(t)\right]=\frac{1}{3} S_{i k j}(t)-\frac{1}{3} D_{i k j}(t)
\end{aligned}
$$

where $r_{i j k}(t)$ denotes the fluorescence emission anisotropy and with

$$
\begin{aligned}
& S_{i j k}(t)=3 \mathbf{c}_{j, 00} \exp \left(\mathbf{A}_{k, 00} t\right) \mathbf{b}_{i k, 00} \\
& D_{i j k}(t)=3 \mathbf{c}_{j, 2 M} \exp \left(\mathbf{A}_{D k} t\right) \mathbf{b}_{i k, 2 M}
\end{aligned}
$$

As before, the subscripts $i, j$, and $k$ in $I_{\| i j k}(t)$ and $I_{\perp i j k}(t)$ [Eq. (48)], in $S_{i j k}(t)$ [Eq. (49)] and in $D_{i j k}(t)$ [Eq. (50)] refer to $\lambda_{i}^{\text {ex }}, \lambda_{j}^{\mathrm{em}}$, and $[\mathrm{X}]_{k}$, respectively. $S_{i j k}(t)$ corresponds to the total time-resolved emission of the photophysical system, is independent of rotational diffusion, and does not contain any information about the orientations of the transition moments. Information about rotational diffusion is contained exclusively in $D_{i j k}(t)$.

Matrix $\mathbf{A}_{k, 00}$ in Eq. (49) is identical with $\mathbf{A}_{k}$ defined by Eq. (26). $\mathbf{A}_{D k}$ in Eq. (50) is defined as:

$$
\mathbf{A}_{D k}=\left(\begin{array}{ccccc}
\mathbf{A}_{D k, 2-2} & 0 & 0 & 0 & 0 \\
0 & \mathbf{A}_{D k, 2-1} & 0 & 0 & 0 \\
0 & 0 & \mathbf{A}_{D k, 20} & 0 & 0 \\
0 & 0 & 0 & \mathbf{A}_{D k, 21} & 0 \\
0 & 0 & 0 & 0 & \mathbf{A}_{D k, 22}
\end{array}\right)
$$

with blocks $\mathbf{A}_{D k, 2 M}$ given by:

$$
\mathbf{A}_{D k, 2 M}=\left(\begin{array}{cc}
-\left(k_{01}+k_{21}[\mathrm{X}]_{k}+D_{1,2 M}\right) & k_{12} \\
k_{21}[\mathrm{X}]_{k} & -\left(k_{02}+k_{12}+D_{2,2 M}\right)
\end{array}\right)
$$


with $M=-2,-1,0,1,2 . D_{n, 2 M}(n$ denotes either 1 or 2$)$ is given by

$$
D_{n, 2 M}=6 D_{\perp n}+M^{2}\left(D_{\| n}-D_{\perp n}\right)
$$

$D_{\perp n}$ and $D_{\| n}$ (see Figure 1) are the components of the rotational diffusion tensor of the cylindrically symmetric species $n$ in its molecular reference frame $(x, y, z)$, chosen such that the rotational diffusion tensor is diagonal, ${ }^{12}$ reducing to the unique component $D_{n}$ (= $\left.D_{\perp n}=D_{\| n}\right)$ in the case of the spherically symmetric rotor $n$.

For a spherically symmetric rotor $\left(D_{n}=D_{\perp n}=D_{\| n}\right)$, the matrices $\mathbf{A}_{D k, 2 M}$ [Eq. (52)] are all identical and independent of $M$. In this case matrix block $\mathbf{A}_{D k, 2 M}$ can be written as:

$$
\mathbf{A}_{D k, 2 M}=\left(\begin{array}{cc}
-\left(k_{01}+k_{21}[\mathrm{X}]_{k}+6 D_{1}\right) & k_{12} \\
k_{21}[\mathrm{X}]_{k} & -\left(k_{02}+k_{12}+6 D_{2}\right)
\end{array}\right)
$$

The elements of vector $\mathbf{b}_{i k, L M}\{$ with $L=M=0$ [Eq. (49)], or $L=2$ and $M= \pm 2, \pm 1,0$ [Eq. (50)]\} are the excitation coefficients $b_{n i k, L M}$. In $b_{n i k, L M} n$ stands for either species 1 or 2 ; $i$ and $k$ refer to $\lambda_{i}^{e x}$ and $[\mathrm{X}]_{k}$, respectively; $L$ and $M$ refer to the orientation of the absorption transitions. The elements $b_{n i k, L M}$ can be expressed as the product of the initial concentration of $n^{*}, b_{n i k}=\left[n^{*}\right]_{0}$, the appropriate spherical harmonic $Y_{L}^{M}\left(\hat{\mathbf{a}}_{n}\right)^{18}$ for the orientation of the absorption transition moment $\hat{\mathbf{a}}_{n}$ in the molecular frame of species $n$, and a scaling factor $B_{L}$ :

$$
b_{n i k, L M}=B_{L} b_{n i k} Y_{L}^{M}\left(\hat{\mathbf{a}}_{n}\right)
$$

with $B_{0}=\frac{1}{12} \sqrt{\frac{1}{\pi^{3}}}$ and $B_{2}=\frac{1}{30} \sqrt{\frac{5}{\pi^{3}}}$.

For $L=M=0$, we have $Y_{0}^{0}\left(\hat{\mathbf{a}}_{n}\right)=\frac{1}{\sqrt{4 \pi}}$ and $b_{n i k, 00}=\frac{b_{n i k}}{24 \pi^{2}}$.

The $2 \times 1$ vector $\mathbf{b}_{i k, 00}$ in Eq. (49) is explicitly given by: 


$$
\mathbf{b}_{i k, 00}=\left(b_{1 i k, 00} \quad b_{2 i k, 00}\right)^{\mathrm{T}}
$$

whereas the $10 \times 1$ vector $\mathbf{b}_{i k, 2 M}$ in Eq. (50) is expressed as:

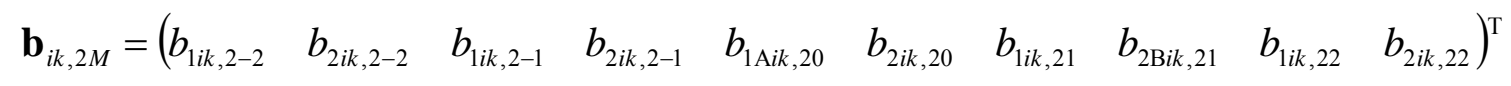

The elements of vector $\mathbf{c}_{j, L M}$ \{with $L=M=0$ [Eq. (49)], or $L=2$ and $M= \pm 2, \pm 1,0$ [Eq. (50)]\} are the corresponding emission coefficients $C_{m j, L M}\left(m\right.$ represents either species $1^{*}$ or $2^{*}$ ). The emission coefficients $C_{m j, L M}$ are given by:

$$
c_{m j, L M}=C_{L} c_{m j} Y_{L}^{M^{*}}\left(\hat{\mathbf{e}}_{m}\right)
$$

where $C_{0}=\frac{16}{3} \sqrt{\pi^{5}}, C_{2}=\frac{16}{15} \sqrt{\frac{\pi^{5}}{5}}$, and $Y_{L}^{M^{*}}\left(\hat{\mathbf{e}}_{m}\right)$ is the complex conjugate of the appropriate spherical harmonic for the orientation of the emission transition moment $\hat{\mathbf{e}}_{m}$ in the molecular frame.

For $L=M=0$, we have $c_{m j, 00}=\frac{8 \pi^{2} c_{m j}}{3}$.

The coefficient $c_{m j}$ is defined by Eq. (9). Vector $\mathbf{c}_{j, 00}$ in Eq. (49) is explicitly given by:

$$
\mathbf{c}_{j, 00}=\left(\begin{array}{ll}
c_{1 j, 00} & c_{2 j, 00}
\end{array}\right)
$$

while vector $\mathbf{c}_{j, 2 M}$ in Eq. (50) is expressed as:

$$
\mathbf{c}_{j, 2 M}=\left(\begin{array}{llllllllll}
c_{1 j, 2-2} & c_{2 j, 2-2} & c_{1 j, 2-1} & c_{2 j, 2-1} & c_{1 j, 20} & c_{2 j, 20} & c_{1 j, 21} & c_{2 j, 21} & c_{1 j, 22} & c_{2 j, 22}
\end{array}\right)
$$

Since both $S_{i j k}(t)=I_{\| i j k}(t)+2 I_{\perp i j k}(t)$ [Eq. (49)] and $D_{i j k}(t)=I_{|| j i k}(t)-I_{\perp i j k}(t)$ [Eq. (50)] can be expressed in matrix form, the identification analysis via similarity transformation is carried out using the $S_{i j k}(t)$ and $D_{i j k}(t)$ functions. 


\section{Kinetic parameters}

We start first with the identification via similarity transformation involving $S_{i j k}(t)$. For $f(t$, $\mathbf{A}, \mathbf{b}, \mathbf{c})=S_{i j k}\left(t, \mathbf{A}_{k, 00}, \mathbf{b}_{i k, 00}, \mathbf{c}_{j, 00}\right), \mathbf{A}_{k, 00}$ in Eq. (49) is identical with $\mathbf{A}_{k}$ defined by Eq. (26), $\mathbf{b}_{i k, 00}$ and $\mathbf{c}_{j, 00}$ are given by Eqs. (56) and (59), respectively, and $\mathbf{T}$ is defined by Eq. (33). As $S_{i j k}(t)$ reflects the time dependence of the total fluorescence and contains information only on the excited states, the identifiability analysis will be the same as that derived for a

reversible intermolecular two-state excited-state process without external quencher ${ }^{9}, 10$ (Section 4A). As shown previously, two sets of rate constants are obtained: set I contains the original rate constants: $k_{01}^{+}=k_{01}, k_{12}^{+}=k_{12}, k_{21}^{+}=k_{21}, k_{02}^{+}=k_{02}$ with $\mathbf{T}=t_{1} \mathbf{I}$; set II is the alternative set: $k_{01}^{+}=k_{02}+k_{12}, k_{12}^{+}=k_{01}-k_{02}, k_{21}^{+}=k_{21}, k_{02}^{+}=k_{02}$ with $\mathbf{T}$ given by Eq. (34), provided that $k_{01}>k_{02}$.

Now we consider the identification involving $f(t, \mathbf{A}, \mathbf{b}, \mathbf{c})=D_{i j k}\left(t, \mathbf{A}_{D k}, \mathbf{b}_{i k, 2 M}, \mathbf{c}_{j, 2 M}\right)$ in which we will use the results of the identifiability analysis involving $S_{i j k}(t)$. We assume that the similarity transformations for $S_{i j k}(t)$ and $D_{i j k}(t)$ are independent.

Kinetic parameters of the cylindrically symmetric rotor

Now $\mathbf{A}_{D k}$ is defined by Eq. (51) with blocks $\mathbf{A}_{D k, 2 M}$ given by Eq. (52). $\mathbf{b}_{i k, 2 M}$ is given by Eq. (57) with $b_{n i k, 2 M}$ defined by Eq. (55), $\mathbf{c}_{j, 2 M}$ is given by Eq. (60) with $c_{m j, 2 M}$ defined by Eq. (58). Also the transformations of the various blocks $\mathbf{A}_{D k, 2 M}$ in $\mathbf{A}_{D k}$ [Eq. (51)] are independent of each other. Matrix $\mathbf{T}$ is a block-diagonal matrix:

$$
\mathbf{T}=\left[\begin{array}{ccccc}
\mathbf{T}_{-2} & 0 & 0 & 0 & 0 \\
0 & \mathbf{T}_{-1} & 0 & 0 & 0 \\
0 & 0 & \mathbf{T}_{0} & 0 & 0 \\
0 & 0 & 0 & \mathbf{T}_{1} & 0 \\
0 & 0 & 0 & 0 & \mathbf{T}_{2}
\end{array}\right]
$$


with the matrices $\mathbf{T}_{M}(M=-2,-1,0,1,2)$ expressed as

$$
\mathbf{T}_{M}=\left[\begin{array}{ll}
t_{M, 1} & t_{M, 2} \\
t_{M, 3} & t_{M, 4}
\end{array}\right]
$$

Because $\mathbf{T}$ and $\mathbf{A}_{D k}$ are both block-diagonal matrices, the matrix multiplication of Eq. (15) is split into five separate matrix multiplications (two of those are identical; $M=-2$ and $M=+2 ; M=-1$ and $M=+1)$. It is straightforward to show that the matrix multiplication [Eq. (15)] involving $\mathbf{A}_{D k, 2 M}^{+}$and $\mathbf{A}_{D k, 2 M}[$ for $M= \pm 2, \pm 1,0]$ leads to two sets of parameters: set III $\quad\left(k_{01}^{+}+D_{1,2 M}^{+}=k_{01}+D_{1,2 M}, \quad k_{12}^{+}=k_{12}, \quad k_{21}^{+}=k_{21}\right.$, $\left.k_{02}^{+}+D_{2,2 M}^{+}=k_{02}+D_{2,2 M}\right) \quad$ corresponding to $\quad \mathbf{T}_{M}=t_{M, 1} \quad \mathbf{I}$, and set IV $\left(k_{01}^{+}+D_{1,2 M}^{+}=k_{02}+k_{12}+D_{2,2 M}, \quad k_{12}^{+}=k_{01}-k_{02}+D_{1,2 M}-D_{2,2 M}, \quad k_{21}^{+}=k_{21}\right.$, $\left.k_{02}^{+}+D_{2,2 M}^{+}=k_{02}+D_{2,2 M}\right) \quad$ corresponding to $\mathbf{T}_{M}$ given by Eq. (63) with $t_{M, 3} / t_{M, 1}=\left(k_{01}-k_{02}-k_{12}\right) / k_{12}:$

$$
\mathbf{T}_{M}=\left[\begin{array}{cc}
t_{M, 1} & 0 \\
t_{M, 3} & t_{M, 1}+t_{M, 3}
\end{array}\right]
$$

To solve for the individual $k_{01}^{+}, k_{12}^{+}, k_{21}^{+}, k_{02}^{+}, D_{\perp 1}^{+}, D_{\| 1}^{+}, D_{\perp 2}^{+}$, and $D_{\| 2}^{+}$, one should combine the equations describing sets I and II with the equations describing sets III and IV. This produces two possible solutions. (1) The original set I of rate constants is combined with the original set of rotational diffusion coefficients: $D_{\perp 1}^{+}=D_{\perp 1}, D_{\| 1}^{+}=D_{\| 1}$, $D_{\perp 2}^{+}=D_{\perp 2}, D_{\| 2}^{+}=D_{\| 2}$. (2) If the rotational diffusion coefficients of both species are equal ( $D_{\perp 1}=D_{\perp 2}$ and $D_{\| 1}=D_{\| 2}$ ), an alternative set of rate constants (i.e., set II of Section 4A) 
and the true rotational diffusion coefficients $\left(D_{\perp 1}^{+}=D_{\perp 2}^{+}=D_{\perp 1}=D_{\perp 2} \quad\right.$ and $\left.D_{\| 1}^{+}=D_{\| 2}^{+}=D_{\| 1}=D_{\| 2}\right)$ may be obtained.

The same result can be obtained by considering the elementary functions $\sigma_{n}$ [Eq. (12)] for the matrix blocks $\mathbf{A}_{D k, 2 M}$, i.e. $\sigma_{D 1 k, 2 M}$ and $\sigma_{D 2 k, 2 M}$. The six eigenvalues of $\mathbf{A}_{D k}$ can be arranged into three pairs, each pair corresponding to a different value of $M$, and each pair can be assigned to the proper $M .^{12}$ Therefore, the functions $\sigma_{D 1 k, 2 M}$ and $\sigma_{D 2 k, 2 M}$ can be obtained unambiguously.

It can be shown that

$$
\begin{aligned}
& \sigma_{D 1 k, 2 M}-\sigma_{1 k}=-D_{1,2 M}-D_{2,2 M} \\
& \left(\sigma_{D 2 k, 2 M}-\sigma_{2 k}\right)-\left(\sigma_{D 2 l, 2 M}-\sigma_{2 l}\right)=k_{21}\left([\mathrm{X}]_{k}-[\mathrm{X}]_{l}\right) D_{2,2 M}
\end{aligned}
$$

from which $D_{1,2 M}$ and $D_{2,2 M}$ are immediately recovered. $D_{1,2 M}$ and $D_{2,2 M}$ considered for two values of $M$ then provide the required rotational diffusion coefficients $D_{\perp 1}, D_{\| 1}, D_{\perp 2}$, and $D_{\| 2}$.

To summarize, the identifiability analysis involving both $S_{i j k}(t)$ and $D_{i j k}(t)$ shows that the model for reversible intermolecular two-state excited-state processes with coupled rotational diffusion for a cylindrically symmetric ellipsoid is uniquely identifiable in terms of rate constants and rotational diffusion coefficients when the rotational diffusion of the two species is different. If the rotational characteristics of the two species are identical, a second set of rate constants (set II) is possible. Kinetic parameters of the spherically symmetric rotor An identification analysis based on similarity transformation for a spherically symmetric rotor similar to that for the cylindrically symmetric ellipsoid also gives two solutions. (1) 
If the rotational diffusion coefficients of both species are different $\left(D_{1} \neq D_{2}\right)$, the unique set of true rate constants (i.e., set I of Section 4A) and true rotational diffusion coefficients $\left(D_{1}^{+}=D_{1}, D_{2}^{+}=D_{2}\right)$ are obtained. (2) When the rotational diffusion coefficients of both species are identical $\left(D_{1}=D_{2}\right)$, an alternative set of rate constants (i.e., set II of Section 4A) may be obtained together with the true rotational diffusion coefficients $\left(D_{1}^{+}=D_{2}^{+}=D_{1}=D_{2}\right)$. The same conclusions can be arrived at by considering the elementary functions $\sigma_{n}$ [Eq. (12)] for matrix $\mathbf{A}_{D k, 2 M}$ [Eq. (54)],

$$
\begin{aligned}
& \sigma_{D 1 k}=\sigma_{1 k}-6\left(D_{1}+D_{2}\right) \\
& \sigma_{D 2 k}=\sigma_{2 k}+6\left(k_{01}+k_{21}[X]_{k}\right) D_{2}+6\left(k_{02}+k_{12}\right) D_{1}+36 D_{1} D_{2}
\end{aligned}
$$

and by evaluating these expressions at two concentrations of coreactant X.

\section{Parameters related to excitation and emission}

For $S_{i j k}(\mathrm{t})$, use of normalized $\widetilde{b}_{1 i k, 00}\left(\widetilde{b}_{2 i k, 00}=1-\widetilde{b}_{1 i k, 00}\right)$ and $\widetilde{c}_{1 j}\left(\widetilde{c}_{2 j}=1-\widetilde{c}_{1 j}\right)$ for $\mathbf{T}=t_{1} \mathbf{I}$

leads to unique normalized parameters: $\widetilde{b}_{1 i k, 00}^{+}=\widetilde{b}_{1 i k}$ and $\widetilde{c}_{1 j, 00}^{+}=\widetilde{c}_{1 j} . \widetilde{b}_{1 i k}$ and $\widetilde{c}_{1 j}$ are defined by Eqs. (37) and (38), respectively.

For $D_{i j k}(t)$ and $\mathbf{T}_{M}=t_{M, 1} \mathbf{I}$ (corresponding to set $\mathrm{I}$ and the true rotational diffusion coefficients), one has

$$
b_{n i k, 2 M}^{+} C_{m j, 2 M}^{+}=b_{n i k, 2 M} C_{m j, 2 M}
$$

with $m, n=1,2$ and $M= \pm 2, \pm 1,0$.

The products of the spherical harmonics implicitly contained in Eq. (66) can be summed via the addition theorem, yielding the second-order Legendre polynomial $P_{2}\left(\hat{\mathbf{a}}_{n}\right.$. $\left.\hat{\mathbf{e}}_{m}\right)$ of the cosine of the angle between transition moments $\hat{\mathbf{a}}_{n}$ and $\hat{\mathbf{e}}_{m}$, 


$$
\sum_{M=-2}^{2} b_{n i k, 2 M} C_{m j, 2 M}=\frac{5}{4 \pi} B_{2} C_{2} b_{n i k} C_{m j} P_{2}\left(\hat{\mathbf{a}}_{n} \cdot \hat{\mathbf{e}}_{m}\right)
$$

This theorem in combination with $b_{l i k}^{+} c_{m j}^{+}=b_{l i k} c_{m j}$ yields

$$
P_{2}\left(\hat{\mathbf{a}}_{n}^{+} \cdot \hat{\mathbf{e}}_{m}^{+}\right)=P_{2}\left(\hat{\mathbf{a}}_{n} \cdot \hat{\mathbf{e}}_{m}\right)
$$

with $n, m=1,2$ and where $\hat{\mathbf{a}}_{n}^{+}$and $\hat{\mathbf{e}}_{m}^{+}$denote alternative transition moments. This implies that all $P_{2}\left(\hat{\mathbf{a}}_{n} . \hat{\mathbf{e}}_{m}\right)$ can be uniquely determined.

Use of normalized $\widetilde{b}_{1 i k, 2 M}\left(\widetilde{b}_{2 i k, 2 M}=1-\widetilde{b}_{1 i k, 2 M}\right)$ and $\widetilde{c}_{1 j, 2 M}\left(\widetilde{c}_{2 j, 2 M}=1-\widetilde{c}_{1 j, 2 M}\right)$ for $\mathbf{T}_{M}=$ $t_{M, 1} \mathbf{I}$ leads to unique normalized parameters:

$$
\begin{aligned}
& \tilde{b}_{1 i k, 2 M}^{+}=\widetilde{b}_{1 i k, 2 M} \\
& \widetilde{c}_{1 j, 2 M}^{+}=\widetilde{c}_{1 j, 2 M}
\end{aligned}
$$

Substitution of Eq. (55) in Eq. (69) gives

$$
\frac{Y_{2}^{M}\left(\hat{\mathbf{a}}_{1}^{+}\right)}{Y_{2}^{M}\left(\hat{\mathbf{a}}_{2}^{+}\right)}=\frac{Y_{2}^{M}\left(\hat{\mathbf{a}}_{1}\right)}{Y_{2}^{M}\left(\hat{\mathbf{a}}_{2}\right)}
$$

so that the ratio of the spherical harmonics for the orientation of the absorption transition moments $\hat{\mathbf{a}}_{1}$ and $\hat{\mathbf{a}}_{2}$ is uniquely identified.

Similarly, substitution of Eq. (58) in Eq. (70) yields

$$
\frac{Y_{2}^{M^{*}}\left(\hat{\mathbf{e}}_{1}^{+}\right)}{Y_{2}^{M^{*}}\left(\hat{\mathbf{e}}_{2}^{+}\right)}=\frac{Y_{2}^{M^{*}}\left(\hat{\mathbf{e}}_{1}\right)}{Y_{2}^{M^{*}}\left(\hat{\mathbf{e}}_{2}\right)}
$$

implying that the ratio of the spherical harmonics for the orientation of the emission transition moments $\hat{\mathbf{e}}_{1}$ and $\hat{\mathbf{e}}_{2}$ is uniquely determined.

Information about the relative orientation of the transition moments can also obtained from the Markov parameters $m_{D n, i k j}$. On introducing the scaling factor $\kappa_{i j k}$, one has that 


$$
m_{D n, i k j}=\frac{8 \pi}{25} \kappa_{i k j} \sum_{M=0}^{2} \sum_{l, m=1}^{2}\left[\mathbf{A}_{D k, 2 M}{ }^{n}\right]_{l m} \widetilde{b}_{l i k} \widetilde{c}_{m j} \Delta_{l m, 2 M}
$$

where $\Delta_{l m, 2 M}$ are geometrical factors given, for $M=0$, by

$$
\Delta_{l m, 20}=Y_{20}^{*}\left(\hat{\mathbf{e}}_{m}\right) Y_{20}\left(\hat{\mathbf{a}}_{l}\right)
$$

and, for $M=1,2$ :

$$
\Delta_{l m, 2 M}=Y_{2-M}^{*}\left(\hat{\mathbf{e}}_{m}\right) Y_{2-M}\left(\hat{\mathbf{a}}_{l}\right)+Y_{2 M}^{*}\left(\hat{\mathbf{e}}_{m}\right) Y_{2 M}\left(\hat{\mathbf{a}}_{l}\right)
$$

Since the $m_{D n, i k j}$ are all linear in $\Delta_{l m, 2 M}$, and since $\widetilde{b}_{l i k}, \widetilde{c}_{m j}$, the rate constants, $D_{\perp l}$, and $D_{\| l}$ are already determined, $\Delta_{l m, 2 M}$ can readily be found from the set $m_{D n, i k j}$, where $n=0$, $1, \ldots, 5$, considered for two excitation wavelengths $\lambda_{i}^{\text {ex }}$ in the same absorption band or for two values of the coreactant concentration $[\mathrm{X}]$. Since, according to the addition theorem,

$$
P_{2}\left(\hat{\mathbf{a}}_{l} \cdot \hat{\mathbf{e}}_{m}\right)=\frac{4 \pi}{5} \sum_{M=0}^{2} \Delta_{l m, 2 M}
$$

the angles between all pairs $\hat{\mathbf{a}}_{l}$ and $\hat{\mathbf{e}}_{m}$ can be determined.

In conclusion, if the rotational diffusion coefficients of the two rotors are different, rotational diffusion coupled to a reversible intermolecular two-state excited-state process makes this model uniquely identifiable in terms of rate constants, rotational diffusion constants and normalized $\widetilde{b}_{1 i k, 2 M}$ and $\widetilde{c}_{2 j, 2 M}$.

\section{Discussion and conclusions}

In this review we have shown that the compartmental analysis approach is excellently suited to describe the kinetics and identifiability of models frequently encountered in photophysics. The term "compartment" in a photophysical context is defined as a subsystem composed of a distinct type of species that acts kinetically in a unique way. 
The compartmental systems considered here consist of one excited-state compartment, two reversibly interconnected excited-state compartments, and their corresponding ground-state compartments. Three models of reversible intermolecular two-state excitedstate processes in isotropic environments are discussed: (1) model without external quencher, (2) model with added quencher, (3) model with coupled species-dependent rotational diffusion described by Brownian reorientation. In a first step, we applied the similarity transformation approach to provide us with the explicit relationships between the true and alternative model parameters. The other identifiability approach based on Markov parameters and elementary functions $\sigma_{1,2}$ of the eigenvalues $\gamma_{1,2}$ was subsequently used to find the experimental conditions under which the model parameters can be recovered. Global compartmental analysis with linked model parameters can be used to estimate these parameters.

Since the deterministic identification analysis assumes by its very own nature that the decay data are error-free (the analysis is based on algebraic equations), the analysis gives the necessary and sufficient requirements to extract the model parameters. However, in real experiments, where the decays do have systematic errors and different parameter sets can sometimes produce fits of similar quality, it is advantageous to collect and analyze more fluorescence decay curves than is required according to the deterministic identification.

Finally, numerical identifiability via global compartmental analysis ${ }^{19}$ has been used to estimate rate constants and parameters related to excitation and emission for a number of reversible intermolecular two-state excited-state processes using fluorescence decay surfaces measured at various excitation and emission wavelengths and coreactant 
concentrations. Global compartmental analysis has the advantage that the parameters of interest are determined directly from the complete decay data surface in a single step. Applications of global compartmental analysis of reversible intermolecular two-state excited-state processes comprise excimer formation of pyrene, ${ }^{20}$ exciplex formation between 1-methylpyrene and trietheylamine, ${ }^{21}$ photophysics of 2-naphthol, ${ }^{22}$ photophysics of the binding of $\mathrm{Ca}^{2+}$ by Fura- $2,{ }^{23}$ Quin- $2,{ }^{24}$ APTRA-BTC, ${ }^{25}$ and Thio$\mathrm{H},{ }^{26}$ photophysics of the fluorescent $\mathrm{K}^{+}$indicator $\mathrm{PBFI},{ }^{27}$ the fluorescent probe $\mathrm{SBFO}^{28}$ for $\mathrm{Na}^{+}$and Mag-fura- $2^{29}$ for $\mathrm{Mg}^{2+}$.

\section{Acknowledgments}

Special thanks are due to all our coworkers who have contributed to the development of the compartmental modeling approach in photophysics. NB thanks Prof. F. C. De Schryver (K.U.Leuven) for his interest and support. The Fonds voor Wetenschappelijk Onderzoek - Vlaanderen (FWO) and DWTC (Belgium) through IAP-V-03 are acknowledged for continuing support. 

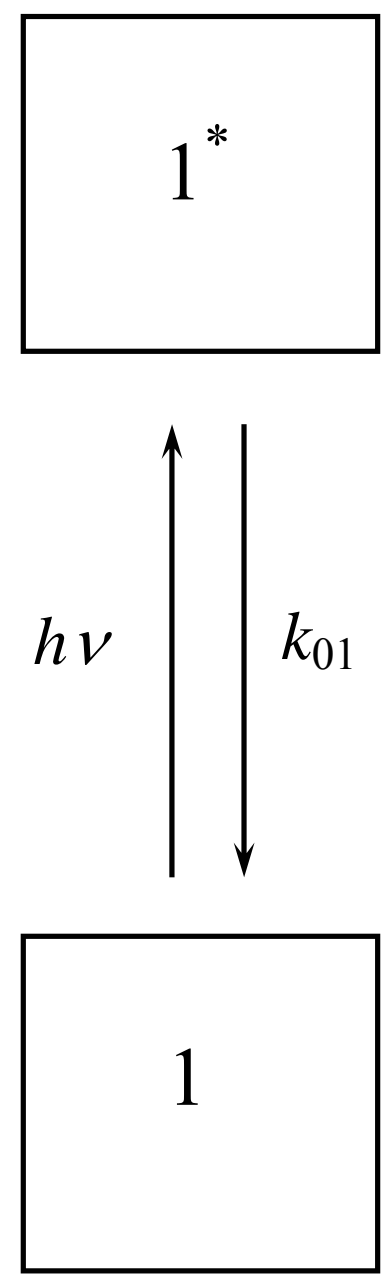

Scheme 1. Scheme representing a one-state excited-state process described by the deactivation rate constant $k_{01}$. 


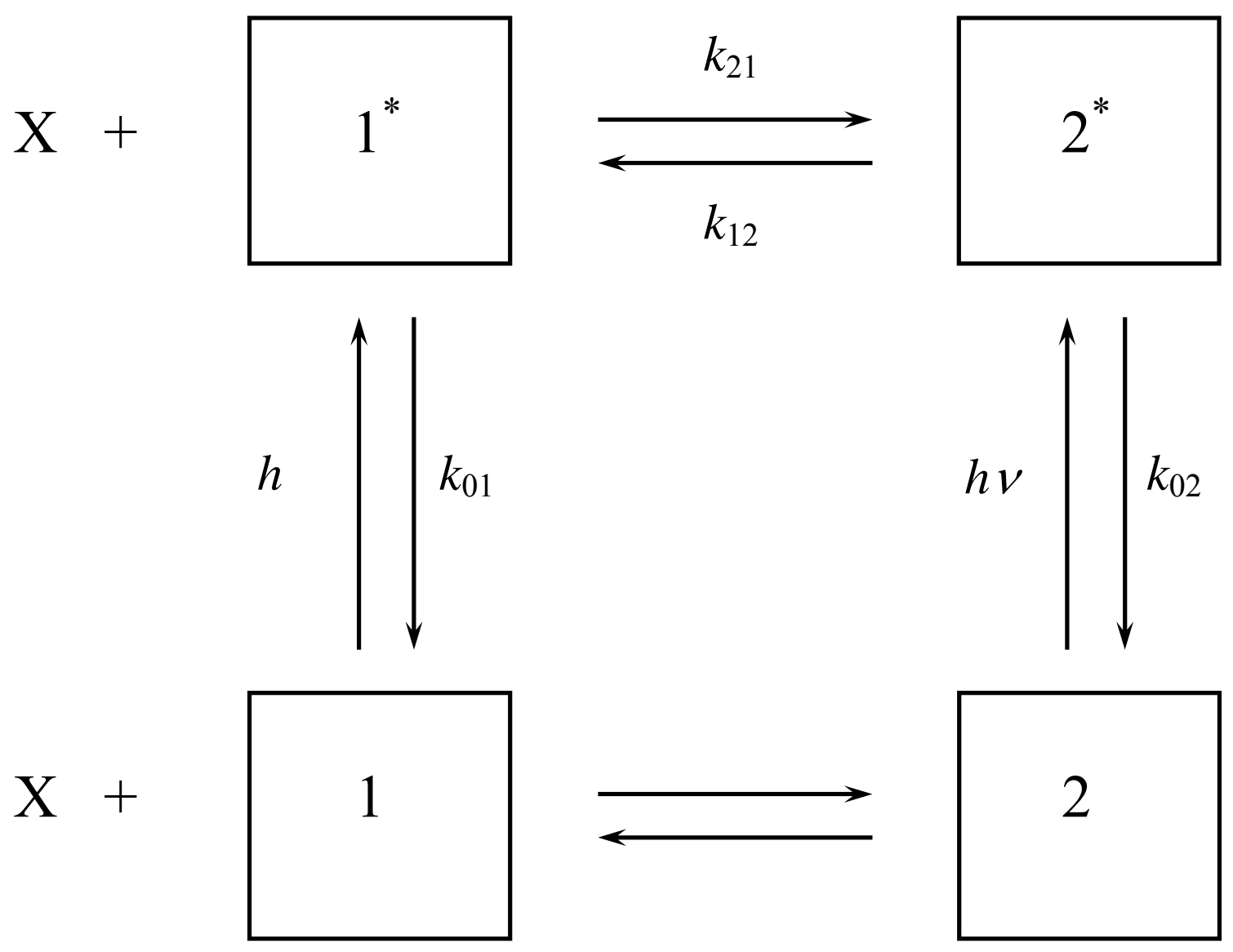

Scheme 2. Scheme representing a reversible intermolecular two-state excited-state process. The excited-state processes are described by the deactivation rate constants $k_{01}$ and $k_{02}$, and the excited-state exchange rate constants $k_{21}$ and $k_{12}$. The transformation of species 1 and $1^{*}$ into, respectively, 2 and $2^{*}$ is mediated by coreactant $\mathrm{X}$. 


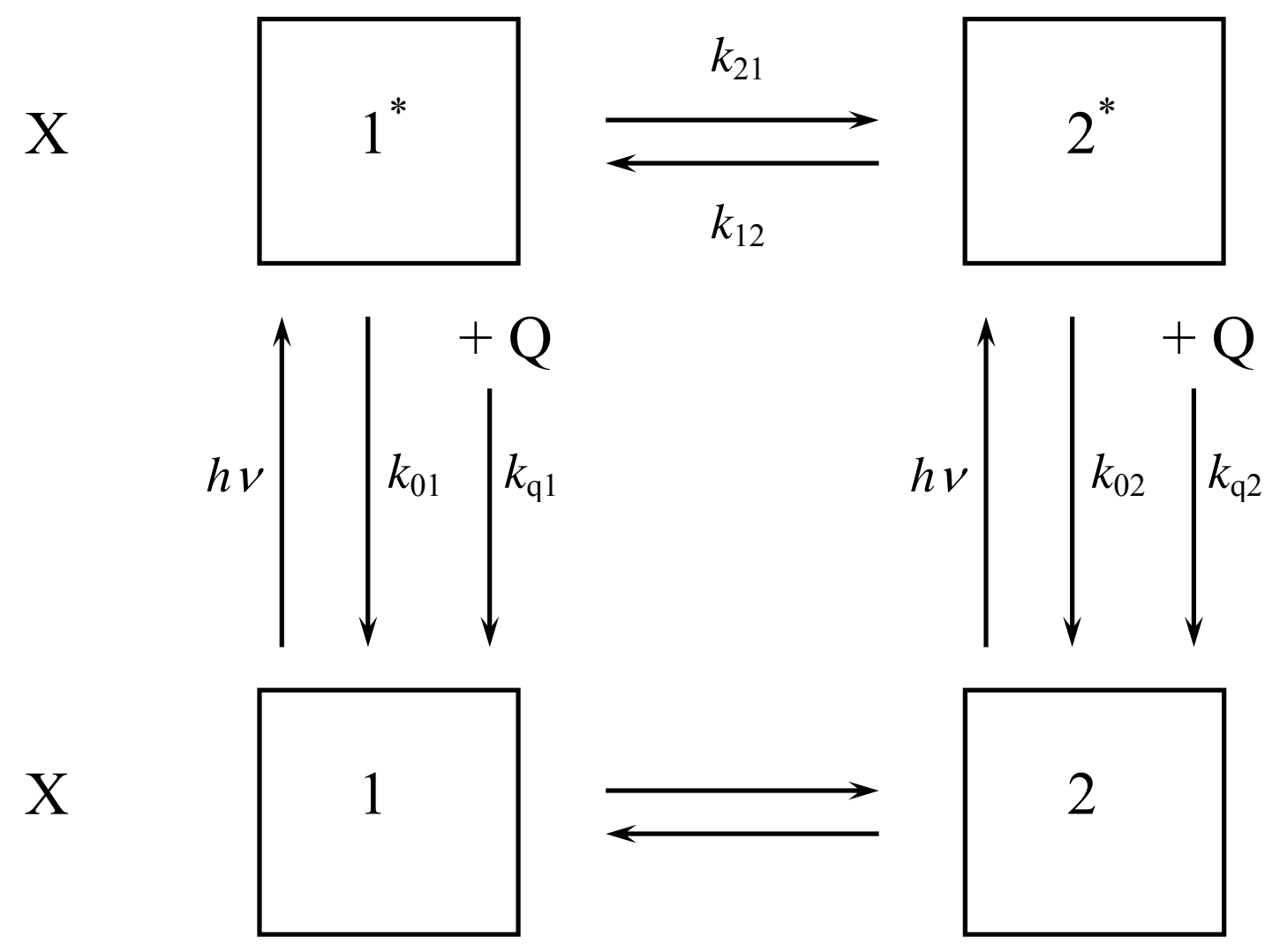

Scheme 3. Scheme representing a reversible intermolecular two-state excited-state process with external quencher. It is assumed that the quencher $\mathrm{Q}$ has only an effect on the excited species and does not affect the ground-state equilibrium. The excited-state processes are described by the deactivation rate constants $k_{01}$ and $k_{02}$, and the excitedstate exchange rate constants $k_{21}$ and $k_{12}$ as in Scheme 2. The transformation of species 1 and $1^{*}$ into, respectively, 2 and $2^{*}$ is mediated by coreactant $\mathrm{X}$. The additional deactivation of $1^{*}$ and $2^{*}$ due to the external quencher $Q$ is described by the rate constants $k_{\mathrm{q} 1}$ and $k_{\mathrm{q} 2}$, respectively. 


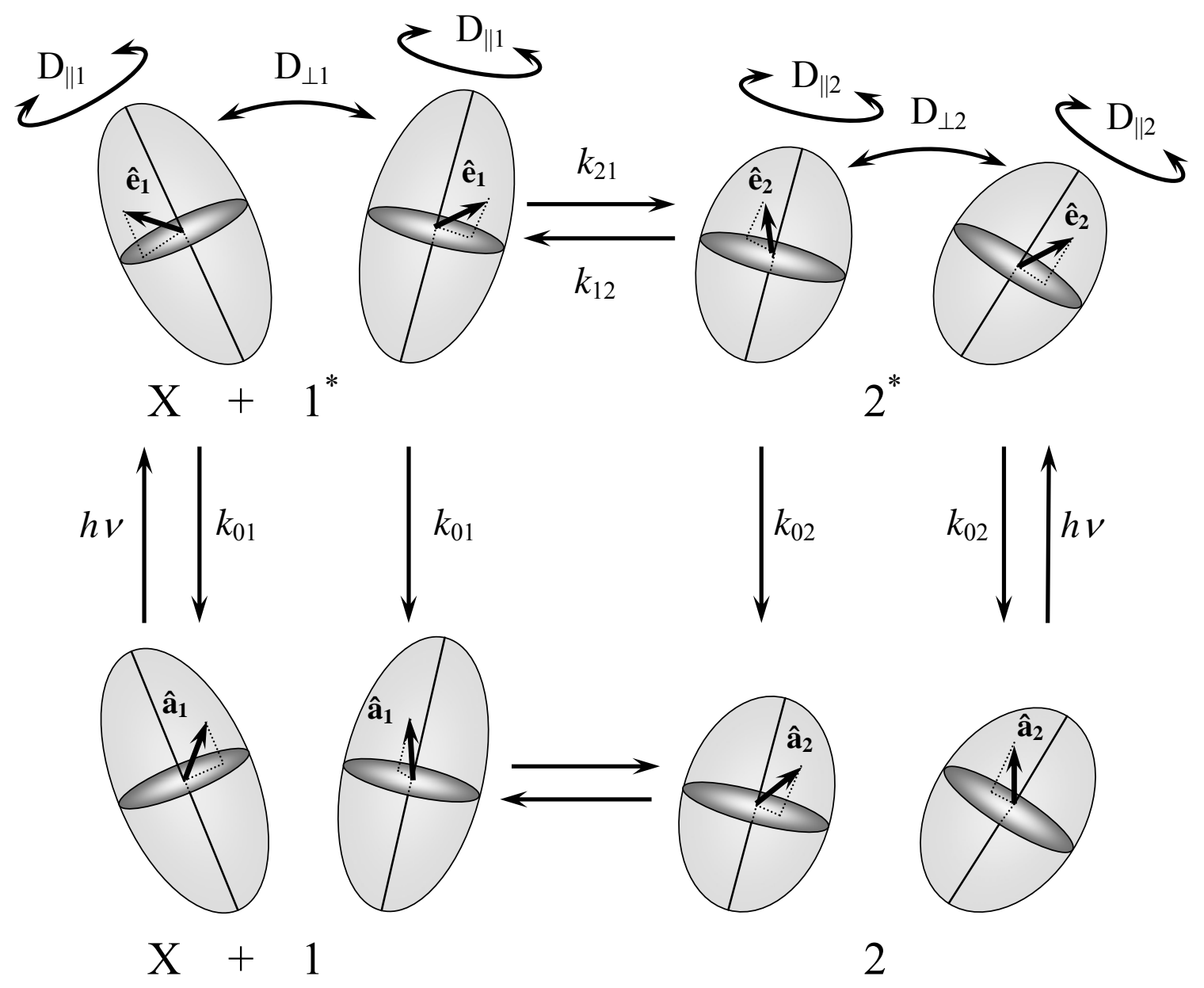

Figure 1. Graphic representation of a reversible intermolecular two-state excited-state process, including rotation. Species $1^{*}$ and $2^{*}$ are pictured as being initially excited from their ground states 1 and 2 by a short linearly polarized light pulse at wavelength $\lambda_{i}^{\text {ex }}$ in a unique absorption band. The excited-state processes are described by the deactivation rate constants $k_{01}$ and $k_{02}$, and the excited-state exchange rate constants $k_{21}$ and $k_{12}$ as in Scheme 2. The transformation of species 1 and $1^{*}$ into, respectively, 2 and $2^{*}$ is mediated by co-reactant X.. Simultaneously the species rotate with rate constants determined by the corresponding rotational diffusion tensors which may differ between the species. The polarized emission of each species depends on the relative orientation of its emission 
transition moment (with unit vector $\hat{\mathbf{e}}_{1}$ or $\hat{\mathbf{e}}_{2}$ ) at the instance of emission with respect to the absorption moment (with unit vector $\hat{\mathbf{a}}_{1}$ or $\hat{\mathbf{a}}_{2}$ ) in the species initially excited. 


\section{References}

[1] J. A. Jacquez, Compartmental Analysis in Biology and Medicine, 3rd Ed., BioMedware, Ann Arbor, 1996.

[2] K. Godfrey, Compartmental Models and Their Application, Academic Press, New York, 1983.

[3] D. H. Anderson, Compartmental Modeling and Tracer Kinetics, in Lecture Notes in Biomathematics, Vol. 50, Springer-Verlag, Berlin, 1983.

[4] J. M. Beechem, M. Ameloot, L. Brand, Chem. Phys. Lett. 1985, 120, 466-472.

[5] J. M. Beechem, M. Ameloot, L. Brand, Anal. Instrum. 1985, 14, 379-402.

[6] M. Ameloot, J. M. Beechem, L. Brand, Chem. Phys. Lett. 1986, 129, 211-219.

[7] K. R. Naqvi, Chem. Phys. Lett. 1987, 136, 407-412.

[8] D.W. Piston, T. Bilash, E. Gratton, J. Phys. Chem. 1989, 93, 3963-3967.

[9] N. Boens, J. Szubiakowski, E. Novikov, M. Ameloot, J. Chem. Phys. 2000, 112, 8260-8266.

[10] M. Ameloot, N. Boens, R. Andriessen, V. Van den Bergh, F. C. De Schryver, J. Phys. Chem. 1991, 95, 2041-2047.

[11] R. Andriessen, M. Ameloot, N. Boens, F.C. De Schryver, J. Phys. Chem. 1992, 96, 314-326.

[12] J. P. Szubiakowski, R. E. Dale, N. Boens, M. Ameloot, J. Chem. Phys. 2004, 121, 7829-7839.

[13] N. Boens, E. Novikov, M. Ameloot, J. Phys. Chem. A, submitted.

[14] R. Bellman, Introduction to Matrix Analysis, McGraw Hill, New York, 1960.

[15] S. Vajda, H. Rabitz, J. Phys. Chem. 1988, 92, 701-707.

[16] M. J. Chappell, K. R. Godfrey, S. Vajda, Math. Biosci. 1990, 102, 41-73.

[17] J. R. Lakowicz, Principles of Fluorescence Spectroscopy, 2nd Ed., Kluwer Academic/Plenum Publishers, New York, 1999.

[18] G. Arfken, Mathematical Methods for Physicists, 3rd Ed., Academic Press, San Diego, 1985.

[19] A general global analysis program using Gaussian-weighted nonlinear least-squares fitting based on Marquardt-Levenberg minimization has been developed jointly by the Division of Photochemistry and Spectroscopy of the K.U.Leuven (Leuven, Belgium) and the Technology Institute of the Belarusian State 
University (Minsk, Belarus). For information about conditions of availability, contact Prof. F. C. De Schryver at Frans.DeSchryver@chem.kuleuven.be.

[20] R. Andriessen, N. Boens, M. Ameloot, F. C. De Schryver, J. Phys. Chem. 1991, 95, 2047-2058.

[21] M. M. H. Khalil, N. Boens, M. Van der Auweraer, M. Ameloot, R. Andriessen, J. Hofkens, F. C. De Schryver, Phys. Chem. 1991, 95, 9375-9381.

[22] V. Van den Bergh, N. Boens, F. C. De Schryver, M. Ameloot, J. Gallay, A. Kowalczyk, Chem. Phys. 1992, 166, 249-258.

[23] V. Van den Bergh, N. Boens, F. C. De Schryver, M. Ameloot, P. Steels, J. Gallay, M. Vincent, A. Kowalczyk, Biophys. J. 1995, 68, 1110-1119.

[24] V. Van den Bergh, N. Boens, F. C. De Schryver, J. Gallay, M. Vincent, Photochem. Photobiol. 1995, $61,442-447$.

[25] N. Boens, E. Cielen, K. Van Werde, F. C. De Schryver, J. Fluorescence 1999, 9, 103-110.

[26] E. Cielen, A. Stobiecka, A. Tahri, G. J. Hoornaert, F. C. De Schryver, J. Gallay, M. Vincent, N. Boens, J. Chem. Soc. Perkin Trans. 2 2002, 1197-1206.

[27] K. Meuwis, N. Boens, F. C. De Schryver, J. Gallay, M. Vincent, Biophys. J. 1995, 68, 2469-2473.

[28] K. Meuwis, N. Boens, F. C. De Schryver, M. Ameloot, J. Gallay, M. Vincent, J. Phys. Chem. B 1998, $102,641-648$.

[29] K. Meuwis, N. Boens, J. Gallay, M. Vincent, Chem. Phys. Lett. 1998, 287, 412-420. 